\title{
Solutions of the three-dimensional radial Dirac equation from the Schrödinger equation with one-dimensional Morse potential
}

\author{
M.G. Garcia ${ }^{\mathrm{a}, \mathrm{b}}$, A.S. de Castro $^{\mathrm{c}}$, P. Alberto ${ }^{\mathrm{d}, *}$, L.B. Castro $^{\mathrm{e}}$ \\ a UNICAMP, Universidade Estadual de Campinas, Departamento de Matemática Aplicada, IMECC, 13081-970, Campinas, SP, Brazil \\ b ITA, Instituto Tecnológico de Aeronáutica, DCTA, 12228-900, São José dos Campos, SP, Brazil \\ c UNESP, Universidade Estadual Paulista, Campus de Guaratinguetá, Departamento de Física e Química, 12516-410, Guaratinguetá, SP, Brazil \\ d CFisUC, University of Coimbra, Physics Department, P-3004-516, Coimbra, Portugal \\ e UFMA, Universidade Federal do Maranhão, Campus Universitário do Bacanga, Departamento de Física, 65080-805, São Luís, MA, Brazil
}

\section{A R T I C L E I N F O}

\section{Article history:}

Received 23 February 2017

Received in revised form 20 April 2017

Accepted 24 April 2017

Available online 27 April 2017

Communicated by P.R. Holland

\section{Keywords:}

Dirac equation

Morse potential

\begin{abstract}
A B S T R A C T
New exact analytical bound-state solutions of the radial Dirac equation in $3+1$ dimensions for two sets of couplings and radial potential functions are obtained via mapping onto the nonrelativistic boundstate solutions of the one-dimensional generalized Morse potential. The eigenfunctions are expressed in terms of generalized Laguerre polynomials, and the eigenenergies are expressed in terms of solutions of equations that can be transformed into polynomial equations. Several analytical results found in the literature, including the Dirac oscillator, are obtained as particular cases of this unified approach.
\end{abstract}

(c) 2017 Elsevier B.V. All rights reserved.

\section{Introduction}

In nonrelativistic quantum mechanics there are several potentials with physical interest that allow for exact solutions, thus offering the possibility of extracting physical information in a way which is not possible otherwise. Among them is the generalized Morse potential $A e^{-\alpha x}+B e^{-2 \alpha x}$ [1-6], the singular harmonic oscillator (SHO) $A x^{2}+B x^{-2}[3,7-22]$, and the singular Coulomb potential (SCP) $A x^{-1}+B x^{-2}[3,7-10,19,21,23-29]$, which have played an important role in atomic, molecular and solid-state physics.

In a recent paper [30], it was shown that nonrelativistic boundstate solutions of the well-known SHO and SCP in arbitrary dimensions can be systematically generated from the nonrelativistic bound states of the one-dimensional generalized Morse potential. The method amounts to a mapping via a Langer transformation [31]. Later, in [32] the method was extended to a modified D-dimensional Klein-Gordon equation featuring a vector interaction nonminimally coupled. That extension of the method used in [30] provided a unified treatment of many known relativistic problems via a mapping onto a unique well-known onedimensional nonrelativistic problem, allowing to obtain exact analytical bound-state solutions for a large class of problems such

\footnotetext{
* Corresponding author.

E-mail addresses: marcelogarcia82@gmail.com (M.G. Garcia), castro@pq.cnpq.br (A.S. de Castro), pedro.alberto@uc.pt (P. Alberto), luis.castro@pq.cnpq.br (L.B. Castro).
}

a vector-scalar SHO plus nonminimal vector Cornell $A x+B x^{-1}$ potentials and nonminimal vector Coulomb (space component) and harmonic oscillator (time component) potentials, vector-scalar Coulomb plus nonminimal vector Cornell potentials and nonminimal vector shifted Coulomb potentials, vector-scalar SCP plus nonminimal vector Coulomb potentials, and also the curious case of a pure nonminimal vector constant potential.

In the present paper, the mapping onto the nonrelativistic bound states of the one-dimensional generalized Morse potential via a Langer transformation is extended to the Dirac equation in $3+1$ dimensions with scalar, vector and tensor radial potentials. This extension allows to obtain exact analytical bound-state solutions for vector-scalar SHO plus tensor Cornell potentials and vector-scalar SCP plus tensor shifted Coulomb potentials. In many cases these represent new solutions, not found before. In all those circumstances the eigenfunctions are expressed in terms of the generalized Laguerre polynomials and the eigenenergies are expressed in terms of irrational equations, which can be cast into polynomial equations. Furthermore, a plethora of results found in the literature obtained through a large variety of methods can now be seen as particular cases of the present method, which is much more straightforward.

The paper is organized as follows. In Sec. 2 we review, as a background, the generalized Morse potential in the Schrödinger equation. The Dirac equation with vector, scalar and tensor couplings and its connection with the generalized Morse potential and the proper form for the potential functions, are presented in Sec. 3 
and two different classes of bound solutions are discussed. The isolated solutions out of the Sturm-Liouville problem are also discussed in this section. In Sec. 4 we draw some conclusions.

\section{Nonrelativistic bound states in a one-dimensional generalized Morse potential}

The time-independent Schrödinger equation is an eigenvalue equation for the characteristic pair $(E, \psi)$ with $E \in \mathbb{R}$. For a particle of mass $M$ embedded in the generalized Morse potential it reads

$\frac{d^{2} \psi(x)}{d x^{2}}+\frac{2 M}{\hbar^{2}}\left(E-V_{1} e^{-\alpha x}-V_{2} e^{-2 \alpha x}\right) \psi(x)=0$,

where $\alpha>0$. Bound-state solutions demand $\int_{-\infty}^{+\infty} d x|\psi|^{2}=1$ and occur only when the generalized Morse potential has a well structure $\left(V_{1}<0\right.$ and $\left.V_{2}>0\right)$. The eigenenergies are given by (see, e.g., $[30,33])$

$E_{n}=-\frac{V_{1}^{2}}{4 V_{2}}\left[1-\frac{\hbar \alpha \sqrt{2 M V_{2}}}{M\left|V_{1}\right|}\left(n+\frac{1}{2}\right)\right]^{2}$,

with

$n=0,1,2, \ldots<\frac{M\left|V_{1}\right|}{\hbar \alpha \sqrt{2 M V_{2}}}-\frac{1}{2}$.

This restriction on $n$ limits the number of allowed states and requires $M\left|V_{1}\right| /\left(\hbar \alpha \sqrt{2 M V_{2}}\right)>1 / 2$ to make the existence of a bound state possible. On the other hand, on making the substitutions

$\hbar \alpha s_{n}=\sqrt{-2 M E_{n}}, \quad \hbar \alpha \xi=2 \sqrt{2 M V_{2}} e^{-\alpha x}$,

the eigenfunctions are expressed as

$\psi_{n}(\xi)=N_{n} \xi^{S_{n}} e^{-\xi / 2} L_{n}^{\left(2 s_{n}\right)}(\xi)$,

where $N_{n}$ are arbitrary constants, and

$L_{n}^{(b)}(x)=\sum_{j=0}^{n} \frac{\Gamma(n+b+1)}{\Gamma(j+b+1)} \frac{(-x)^{j}}{j !(n-j) !}, \quad b>-1$

are the generalized Laguerre polynomials (see, e.g., [34,35]).

\section{The Dirac equation}

The time-independent Dirac equation for a spin $1 / 2$ fermion with energy $\varepsilon$ and with mass $m$, in the presence of a potential reads (with $\hbar=c=1$ )

$(\vec{\alpha} \cdot \vec{p}+\beta m+\mathcal{V}) \Psi=\varepsilon \Psi$,

where $\vec{p}$ is the momentum operator and $\vec{\alpha}$ and $\beta$ are $4 \times 4$ matrices which, in the usual representation, take the form

$\vec{\alpha}=\left(\begin{array}{cc}0 & \vec{\sigma} \\ \vec{\sigma} & 0\end{array}\right), \quad \beta=\left(\begin{array}{cc}I_{2} & 0 \\ 0 & -I_{2}\end{array}\right)$.

Here $\vec{\sigma}$ is a three-vector whose components are the Pauli matrices, and $I_{N}$ stands for the $N \times N$ identity matrix. In the following, we consider

$\mathcal{V}(r)=V_{v}(r)+\beta V_{S}(r)+i \beta \vec{\alpha} \cdot \hat{r} U(r)$.

In the last term, $\hat{r}=\vec{r} / r$, and the radial functions in Eq. (9) are named after the properties their respective terms have under Lorentz transformations: $V_{v}$ corresponds to the time component of a vector potential, $V_{S}$ is a scalar potential, and $U$ is a tensor potential [36]. In spherical coordinates, $\Psi$ is expressed in terms of spinor spherical harmonics
$\Psi(\vec{r})=\left(\begin{array}{c}i \frac{g_{\kappa}(r)}{r} \mathcal{Y}_{\kappa m_{j}}(\hat{r}) \\ -\frac{f_{\kappa}(r)}{r} \mathcal{Y}_{\tilde{\kappa} m_{j}}(\hat{r})\end{array}\right)$,

where $\kappa= \pm(j+1 / 2)=-\tilde{\kappa}$ are eigenvalues of the spin-orbit operator $K=-\beta\left(2 \vec{S} \cdot \vec{L}+I_{4}\right), j$ is the total angular momentum quantum number ( $m_{j}$ refers to its third component), and $\vec{S}$ and $\vec{L}$ are the spin and angular momentum operators, respectively. More explicitly, the spin-orbit coupling quantum number $\kappa$ is related to the upper component orbital angular momentum quantum number $l$ by

$\kappa=\left\{\begin{array}{c}-(l+1)=-(j+1 / 2), \quad j=l+1 / 2(\kappa<0) \\ l=+(j+1 / 2), \quad j=l-1 / 2(\kappa>0) .\end{array}\right.$

The upper and lower radial functions obey the coupled first-order equations:

$\left[\frac{d}{d r}+\frac{\kappa}{r}+U(r)\right] g_{\kappa}(r)=\left[m+\varepsilon-V_{\Delta}(r)\right] f_{\kappa}(r)$

$\left[\frac{d}{d r}-\frac{\kappa}{r}-U(r)\right] f_{\kappa}(r)=\left[m-\varepsilon+V_{\Sigma}(r)\right] g_{\kappa}(r)$,

where we have introduced the "sum" and the "difference" potentials defined by $V_{\Sigma}=V_{v}+V_{s}$ and $V_{\Delta}=V_{v}-V_{s}$.

It is instructive to note that the charge-conjugation operation is accomplished by the changes of sign of $\varepsilon, V_{v}, U$ and $\kappa$. In turn, this means that $V_{\Sigma}$ turns into $-V_{\Delta}, V_{\Delta}$ into $-V_{\Sigma}, g_{\kappa}$ into $f_{\kappa}$ and $f_{\kappa}$ into $g_{\kappa}$. Therefore, to be invariant under charge conjugation, the Dirac equation must contain only a scalar potential. Furthermore, $g_{\kappa}$ and $f_{\kappa}$ should be square-integrable functions for bound states.

Due to charge conjugation, solutions for $V_{\Sigma}=0$ can be conveniently obtained from those ones for $V_{\Delta}=0$, provided those solutions are analytical. These correspond, respectively, to so-called pseudospin and spin symmetry conditions of the Dirac equation (see [37] for a recent review). Therefore, we concentrate our attention to the case $V_{\Delta}=0$. In this case, one obtains a second-order differential equation for $g_{\kappa}$ when $\varepsilon \neq-m$ and a first-order differential equation for $g_{\kappa}$ when $\varepsilon=-m$.

\subsection{The Sturm-Liouville problem for $V_{\Delta}=0(\varepsilon \neq-m)$}

For $V_{\Delta}=0$ and $\varepsilon \neq-m$,

$$
\begin{gathered}
f_{\kappa}(r)=\frac{1}{\varepsilon+m}\left[\frac{d}{d r}+\frac{\kappa}{r}+U(r)\right] g_{\kappa}(r) \\
\frac{d^{2} g_{\kappa}(r)}{d r^{2}}+2 M\left[\widetilde{\varepsilon}-V(r)-\frac{\kappa(\kappa+1)}{2 M r^{2}}\right] g_{\kappa}(r)=0 .
\end{gathered}
$$

The effective energy $\widetilde{\mathcal{E}}$ and the effective potential $V$ are expressed by

$$
2 M \widetilde{\varepsilon}=\varepsilon^{2}-m^{2}
$$

$2 M V(r)=(\varepsilon+m) V_{\Sigma}(r)-\frac{d U(r)}{d r}+2 \kappa \frac{U(r)}{r}+[U(r)]^{2}$,

and $g_{\kappa} \rightarrow 0$ as $r \rightarrow \infty$ for bound-state solutions. The positive parameter $M$ has dimension of mass and no effect on $\varepsilon, V, f_{\kappa}$ and $g_{\kappa}$, and its presence is justified for comparison with eq. (1).

Following Ref. [30], with effective potentials expressed by

$V(r)=A r^{\delta}+\frac{B}{r^{2}}+C$,

the Langer transformation [31]

$g_{\kappa}(r)=\sqrt{r / r_{0}} \phi_{\kappa}(x), \quad r / r_{0}=e^{-\Lambda \alpha x}$, 
with $r_{0}>0, \Lambda>0$ and $\alpha$ being as in eq. (1), transmutes the radial equation (13) into

$$
\begin{aligned}
& \frac{d^{2} \phi_{\kappa}(x)}{d x^{2}}+2 M\left\{-\frac{(\Lambda \alpha S)^{2}}{2 M}-\left(\Lambda \alpha r_{0}\right)^{2}\left[A r_{0}^{\delta} e^{-\Lambda \alpha(\delta+2) x}\right.\right. \\
& \left.\left.+(C-\widetilde{\varepsilon}) e^{-2 \Lambda \alpha x}\right]\right\} \phi_{\kappa}(x)=0
\end{aligned}
$$

with

$S=\sqrt{(\kappa+1 / 2)^{2}+2 M B}$

Comparison of eqs. (17) and (1) shows that for $\delta=0$ or $\delta=-2$, i.e., a pure inversely quadratic potential, bound solutions are not allowed. A connection with the bound states of the generalized Morse potential of eq. (1) is obtained only if the pair $(\delta, \Lambda)$ is equal either to $(2,1 / 2)$ or $(-1,1)$, and, as an immediate consequence of the reality of $S$, i.e., $S^{2}>0$, one must have

$2 M B>-(\kappa+1 / 2)^{2}$.

Actually, if $2 M B>-1 / 4$, the above condition will be satisfied for all values of $\kappa$, so that the term inversely quadratic in (15) cannot be strongly attractive. Furthermore, since the asymptotic behaviour of (17) implies that $\phi_{\kappa}(x) \underset{x \rightarrow+\infty}{\rightarrow} e^{-\Lambda \alpha S x}$ and therefore, from (16), one has

$g_{\kappa}(r) \underset{r \rightarrow 0}{\rightarrow} r^{1 / 2+S}$.

At this point it is already worthy remarking that the fact that i) there are no bound solutions for a pure effective inversely quadratic potential; ii) the determination of the critical strength of the term containing the effective inversely quadratic potential; iii) the boundary condition $g_{\kappa}(0)=0$, all emerge naturally as a consequence of the mapping onto the one-dimensional Morse problem.

Effective potentials with the general form (15) are achieved by choosing the potentials in the Dirac equation as follows

$$
\begin{aligned}
V_{\Sigma}(r) & =\frac{\alpha_{\Sigma}}{r^{2}}+\frac{\beta_{\Sigma}}{r}+\gamma_{\Sigma} r^{2}, \\
U(r) & =\frac{\beta_{u}}{r}+\gamma_{u} r^{\delta_{u}}, \quad \delta_{u}=0 \text { or } 1 .
\end{aligned}
$$

In these last expressions, when $\delta=2$ one must have $\beta_{\Sigma}=0, \delta_{u}=$ 1 and when $\delta=-1$ one has $\gamma_{\Sigma}=0, \delta_{u}=0$.

\subsubsection{The effective singular harmonic oscillator}

With $(\delta, \Lambda)=(2,1 / 2)$ plus the definition $A=M \omega^{2} / 2$, the identification of the bound-state solutions of Eq. (13) with those ones from the generalized Morse potential is done by setting $V_{1}=$ $-\alpha^{2} r_{0}^{2}(\widetilde{\varepsilon}-C) / 4$ and $V_{2}=\alpha^{2} r_{0}^{4} M \omega^{2} / 8$, with $\widetilde{\varepsilon}>C$ and $\omega^{2}>0$, since $V_{1}<0$ and $V_{2}>0$. With $\omega>0$ one can write

$\xi=M \omega r^{2}$.

Furthermore, (3) implies $\widetilde{\varepsilon}>C+\omega(2 n+1)$. Using (2) and (18) one can write the complete solution of the problem as

$$
\begin{aligned}
\widetilde{\varepsilon} & =C+\omega(2 n+1+S) \\
g_{\kappa}(r) & =N r^{1 / 2+S} e^{-M \omega r^{2} / 2} L_{n}^{(S)}\left(M \omega r^{2}\right) .
\end{aligned}
$$

The condition (3) means that

$n \leq\left[\frac{\widetilde{\varepsilon}-C-\omega}{2 \omega}\right]$, where $[x]$ stands for the largest integer less than or equal to $x$. Since $\widetilde{\varepsilon}$ depends quadratically on $\varepsilon$ from eqs. (14) and $\omega$ may depend at most on $\sqrt{\varepsilon}$ (see eq. (26) below), the condition (24) means that there is no limitation on the value of $n$, because it can be as large as the energy can, which in turns means that $n$ in (23) has no upper bound.

Examples of this class of solutions can be reached by choosing

$V_{\Sigma}(r)=\frac{\alpha_{\Sigma}}{r^{2}}+\gamma_{\Sigma} r^{2}, \quad U(r)=\frac{\beta_{u}}{r}+\gamma_{u} r$.

This is the vector-scalar SHO potential plus the tensor potential Cornell potential [38], which under appropriate conditions can describe particular cases like the harmonic oscillator plus a tensor linear potential [39], the harmonic oscillator plus a tensor Cornell potential $[40,41]$, the SHO plus a tensor linear potential [42], the SHO [43,44], the tensor Cornell potential [45] and the Dirac oscillator [46].

The complete identification with the generalized Morse potential is done with the equalities

$$
\begin{aligned}
M \omega & =\sqrt{\gamma_{u}^{2}+\gamma_{\Sigma}(\varepsilon+m)} \\
2 M B & =\left(\beta_{u}+\kappa+1 / 2\right)^{2}-(\kappa+1 / 2)^{2}+\alpha_{\Sigma}(\varepsilon+m) \\
2 M C & =\gamma_{u}\left(2 \beta_{u}+2 \kappa-1\right),
\end{aligned}
$$

which lead, in general, to an irrational equation in $\varepsilon$ :

$$
\begin{aligned}
(\varepsilon+m)( & \varepsilon-m)-\gamma_{u}\left(2 \beta_{u}+2 \kappa-1\right) \\
= & 2(2 n+1+S) \sqrt{\gamma_{u}^{2}+\gamma_{\Sigma}(\varepsilon+m)} \\
= & 2\left(2 n+1+\sqrt{\left(\beta_{u}+\kappa+1 / 2\right)^{2}+\alpha_{\Sigma}(\varepsilon+m)}\right) \\
& \times \sqrt{\gamma_{u}^{2}+\gamma_{\Sigma}(\varepsilon+m)} .
\end{aligned}
$$

We note that if $\alpha_{\Sigma}>0, \gamma_{\Sigma}>0$ and $\beta_{u}=0$, one gets a harmonic oscillator type energy spectrum for positive energy states with $\varepsilon>m$, but there are also states with negative energy, although there would be a minimum value for that energy, because one must have $(\kappa+1 / 2)^{2}+\alpha_{\Sigma}(\varepsilon+m) \geq 0$. If in addition $\alpha_{\Sigma}=0$, one has the (positive energy) generalized relativistic harmonic oscillator with $\gamma_{\Sigma}=1 / 2 m \omega_{1}^{2}, \gamma_{u}=m \omega_{2}$ where $\omega_{1}$ and $\omega_{2}$ are the frequencies defined in [39].

Squaring Eq. (27) successively results into a nonequivalent algebraic equation of degree 8 . Solutions of this algebraic equation that are not solutions of the original equation can be removed by backward substitution. A quartic algebraic equation is obtained when $\alpha_{\Sigma}=0$. For $\alpha_{\Sigma}=\gamma_{u}=0$ one obtains a cubic algebraic equation. However, (27) can be written as a quadratic algebraic equation rendering two branches of solutions symmetrical about $\varepsilon=0$ in the case of a pure tensor Cornell potential $\left(\gamma_{u} \neq 0\right)$ :

$$
\begin{aligned}
\varepsilon & = \pm \sqrt{m^{2}+\gamma_{u}\left(2 \beta_{u}+2 \kappa-1\right)+2\left|\gamma_{u}\right|(2 n+1+S)} \\
& = \pm \sqrt{m^{2}+\gamma_{u}\left(2 \beta_{u}+2 \kappa-1\right)+2\left|\gamma_{u}\right|\left(2 n+1+\left|\beta_{u}+\kappa+1 / 2\right|\right)} .
\end{aligned}
$$

\subsubsection{The effective singular Coulomb potential}

To get the bound states of eq. (13) from those of the generalized Morse potential equation eq. (17) with the pair $(\delta, \Lambda)=(-1,1)$ one must choose $V_{1}=\alpha^{2} r_{0} A$ and $V_{2}=\alpha^{2} r_{0}^{2}(C-\widetilde{\varepsilon})$, with $A<0$ and $\widetilde{\varepsilon}<C$. Now,

$\xi=2 \sqrt{2 M(C-\widetilde{\varepsilon})} r$

and (3) implies $\widetilde{\varepsilon}>C-M A^{2} /\left[2(n+1 / 2)^{2}\right]$. Using (2) and (18) one can write 


$$
\widetilde{\varepsilon}=C-\frac{M A^{2}}{2 \zeta^{2}}
$$

$g_{\kappa}(r)=N r^{1 / 2+S} e^{-M|A| r / \zeta} L_{n}^{(2 S)}\left(\frac{2 M|A| r}{\zeta}\right)$

with

$\zeta=n+1 / 2+S=n+1 / 2+\sqrt{(\kappa+1 / 2)^{2}+2 M B}$.

Again for this class of effective potentials, condition (3) implies that there is no upper bound for $n$. This class of solutions can be obtained by choosing

$V_{\Sigma}(r)=\frac{\alpha_{\Sigma}}{r^{2}}+\frac{\beta_{\Sigma}}{r}, \quad U(r)=\frac{\beta_{u}}{r}+\gamma_{u}$,

which is the vector-scalar SCP plus a shifted Coulomb tensor potential. There results

$$
\begin{aligned}
& 2 M A=\beta_{\Sigma}(\varepsilon+m)+2 \gamma_{u}\left(\beta_{u}+\kappa\right) \\
& 2 M B=\left(\beta_{u}+\kappa+1 / 2\right)^{2}-(\kappa+1 / 2)^{2}+\alpha_{\Sigma}(\varepsilon+m) \\
& 2 M C=\gamma_{u}^{2} .
\end{aligned}
$$

Subject to appropriate constraints, one finds the irrational equation in $\varepsilon$

$$
\begin{aligned}
& (\varepsilon+m)(\varepsilon-m) \\
& =\gamma_{u}^{2}-\left[\frac{2 \gamma_{u}\left(\beta_{u}+\kappa\right)+\beta_{\Sigma}(\varepsilon+m)}{2\left(n+1 / 2+\sqrt{\left(\beta_{u}+\kappa+1 / 2\right)^{2}+\alpha_{\Sigma}(\varepsilon+m)}\right)}\right]^{2} .
\end{aligned}
$$

One example of solutions for these type of radial potentials in the Dirac equation is the Coulomb potential plus a tensor Coulomb potential [47], and the SCP plus a tensor Coulomb potential $[48,49]$.

The very special case $\alpha_{\Sigma}=\gamma_{u}=0$, necessarily with $\beta_{\Sigma}<0$, holds a spectrum given by

$\varepsilon=m \frac{1-\left[\beta_{\Sigma} /(2 \zeta)\right]^{2}}{1+\left[\beta_{\Sigma} /(2 \zeta)\right]^{2}}$,

with $\zeta=n+1 / 2+\left|\beta_{u}+\kappa+1 / 2\right|$. It is interesting that the dependence on the tensor potential parameter $\beta_{u}$ is done only through $\zeta$, which contains the quantity $2 M B$. Therefore, the spectrum is formally similar to the solution of pure $\left(\beta_{u}=0\right)$ Coulomb scalar and vector potentials in spin symmetry conditions [50]. It amounts to have an effective value of $\kappa$, given by $\bar{\kappa}=\kappa+\beta_{u}$.

It is also interesting to see that the special case $\alpha_{\Sigma}=\beta_{\Sigma}=$ $\beta_{u}=0$ gives a spectrum for either spin aligned or spin antialigned, depending on the sign of $\gamma_{u}$.

\subsubsection{Summary of results}

In Table 1 we summarize the conditions for the potential parameters which allow for analytical solutions.

\subsection{Isolated solutions for $V_{\Delta}=0(\varepsilon=-m)$}

We shall now deal with possible solutions for potentials expressed by (21) that can not be expressed by means of the SturmLiouville problem. For $V_{\Delta}=0$ and $\varepsilon=-m$, one can write

$$
\begin{aligned}
& \frac{d g_{\kappa}(r)}{d r}+\left[\frac{\kappa}{r}+U(r)\right] g_{\kappa}(r)=0 \\
& \frac{d f_{\kappa}(r)}{d r}-\left[\frac{\kappa}{r}+U(r)\right] f_{\kappa}(r)=\left[2 m+V_{\Sigma}(r)\right] g_{\kappa}(r),
\end{aligned}
$$

Table 1

General conditions for the potential parameters of eq. (21) in order to have analytical solutions and radial functions $g_{\kappa}(r)$ for $\delta_{u}=1$ (harmonic oscillator type potentials) and for $\delta_{u}=0$ (Coulomb type potentials). $\zeta$ is given by (31).

\begin{tabular}{ll}
\hline$\delta_{u}=1$ & $\delta_{u}=0$ \\
\hline$\beta_{\Sigma}=0$ & $\gamma_{\Sigma}=0$ \\
$M^{2} \omega^{2}=\gamma_{u}^{2}+\gamma_{\Sigma}(\varepsilon+m)>0$ & $2 M A=\beta_{\Sigma}(\varepsilon+m)+2 \gamma_{u}\left(\beta_{u}+\kappa\right)<0$ \\
$S=\left(\beta_{u}+\kappa+1 / 2\right)^{2}+\alpha_{\Sigma}(\varepsilon+m)>0$ & $S=\left(\beta_{u}+\kappa+1 / 2\right)^{2}+\alpha_{\Sigma}(\varepsilon+m)>0$ \\
$g_{\kappa}(r)=N r^{1 / 2+S} e^{-M \omega r^{2} / 2} L_{n}^{(S)}\left(M \omega r^{2}\right)$ & $g_{\kappa}(r)=N r^{1 / 2+S} e^{-M|A| r / \zeta} L_{n}^{(2 S)}\left(\frac{2 M|A| r}{\zeta}\right)$ \\
\hline
\end{tabular}

which arise from (12). Hence,

$g_{\kappa}(r)=N_{g} e^{-v(r)}$,

with

$v(r)=\int^{r} d y\left[\frac{\kappa}{y}+U(y)\right]$.

There is no need to use a lower limit on the integral in (38) because the resulting constant of integration can be lumped in the constant $N_{g}$. On the other hand, the nonhomogeneous differential equation for $f_{\kappa}$ yields the general solution

$f_{\kappa}(r)=\left[N_{f}+N_{g} I(r)\right] e^{+v(r)}$,

where $N_{f}$ is a constant associated to the homogeneous equation for $f_{\kappa}$, and

$I(r)=\int^{r} d y\left[2 m+V_{\Sigma}(y)\right] e^{-2 v(y)}$.

It is also worthwhile to note that this sort of isolated solution can not describe scattering states.

One finds

$v(r)=\ln r^{\left(\beta_{u}+\kappa\right)}+\frac{\gamma_{u}}{\delta_{u}+1} r^{\delta_{u}+1}$.

Because $g_{\kappa}$ and $f_{\kappa}$ are square-integrable functions, $N_{f}=0$ for $\gamma_{u}>0$, and $N_{g}=0$ for $\gamma_{u}<0$. Hence,

$g_{\kappa}(r)=N_{g} r^{-\left(\beta_{u}+\kappa\right)} \exp \left(-\frac{\left|\gamma_{u}\right|}{\delta_{u}+1} r^{\delta_{u}+1}\right)$
$f_{\kappa}(r)=N_{g} I(r) r^{+\left(\beta_{u}+\kappa\right)} \exp \left(+\frac{\left|\gamma_{u}\right|}{\delta_{u}+1} r^{\delta_{u}+1}\right)$,

for $\gamma_{u}>0$, and

$g_{\kappa}(r)=0$

$f_{\kappa}(r)=N_{f} r^{+\left(\beta_{u}+\kappa\right)} \exp \left(-\frac{\left|\gamma_{u}\right|}{\delta_{u}+1} r^{\delta_{u}+1}\right)$,

for $\gamma_{u}<0$.

When $\gamma_{u}>0$, square integrability of $f_{\kappa}$ demands a good behaviour for $I(r)$ at infinity. Calculation shows that

$$
\begin{aligned}
& \left(\delta_{u}+1\right)\left(\frac{2\left|\gamma_{u}\right|}{\delta_{u}+1}\right)^{-2\left(\beta_{u}+\kappa\right) /\left(\delta_{u}+1\right)} I(r) \\
= & 2 m\left(\frac{2\left|\gamma_{u}\right|}{\delta_{u}+1}\right)^{-1 /\left(\delta_{u}+1\right)} \Gamma\left(\frac{1-2\left(\beta_{u}+\kappa\right)}{\delta_{u}+1}, \frac{2\left|\gamma_{u}\right|}{\delta_{u}+1} r^{\delta_{u}+1}\right) \\
& +\alpha_{\Sigma}\left(\frac{2\left|\gamma_{u}\right|}{\delta_{u}+1}\right)^{+1 /\left(\delta_{u}+1\right)} \Gamma\left(-\frac{1+2\left(\beta_{u}+\kappa\right)}{\delta_{u}+1}, \frac{2\left|\gamma_{u}\right|}{\delta_{u}+1} r^{\delta_{u}+1}\right) \\
& +\beta_{\Sigma} \Gamma\left(-\frac{2\left(\beta_{u}+\kappa\right)}{\delta_{u}+1}, \frac{2\left|\gamma_{u}\right|}{\delta_{u}+1} r^{\delta_{u}+1}\right)
\end{aligned}
$$




$$
+\gamma_{\Sigma}\left(\frac{2\left|\gamma_{u}\right|}{\delta_{u}+1}\right)^{-3 /\left(\delta_{u}+1\right)} \Gamma\left(\frac{3-2\left(\beta_{u}+\kappa\right)}{\delta_{u}+1}, \frac{2\left|\gamma_{u}\right|}{\delta_{u}+1} r^{\delta_{u}+1}\right)
$$

where $\Gamma(a, z)$ is the incomplete gamma function [35]

$\Gamma(a, z)=\int_{0}^{z} d t e^{-t} t^{a-1}$.

Due to the behaviour of the integrand near the origin, this integral diverges if Re $a$ is not positive. Furthermore, as $z$ increases $\Gamma(a, z)$ approaches the limiting value $\Gamma(a)$ when $\operatorname{Re} a>0$. Therefore, $I(r)$ diverges if the first argument of the incomplete gamma function of at least one of the terms of $I(r)$ is not positive, and it tends to a constant as $r$ tends to infinity if the first argument of the incomplete gamma function of all the terms of $I(r)$ is positive. For these reasons, $f_{\kappa}$ is not a square-integrable function. An exception, though, occurs when $m=\alpha_{\Sigma}=\beta_{\Sigma}=\gamma_{\Sigma}=0$ just for the reason that $f_{\kappa}$ vanishes identically. Therefore,

$g_{\kappa}(r)=N_{g} r^{-\left(\beta_{u}+\kappa\right)} \exp \left(-\frac{\left|\gamma_{u}\right|}{\delta_{u}+1} r^{\delta_{u}+1}\right)$

$f_{\kappa}(r)=0$,

only for $\gamma_{u}>0$ and $m=V_{\Sigma}=0$.

In addition, a good behaviour of $g_{\kappa}$ and $f_{\kappa}$ near the origin, in the sense of normalization, forces one to the choice $\beta_{u}+\kappa \lessgtr \pm 1 / 2$ for $\gamma_{u} \gtrless 0$.

\section{Concluding remarks}

Based on Ref. [30], we have described a straightforward and efficient procedure for finding a large class of solutions of the Dirac equation in $3+1$ dimensions with radial scalar $V_{s}$ vector $V_{v}$ and tensor $U$ radial potentials, when $V_{s}= \pm V_{v}$, some of which have never been obtained before. Their wave functions are all expressed in terms of generalized Laguerre polynomials and their energy eigenvalues obey analytical equations, either polynomial or irrational which can be cast as polynomial. These include harmonic oscillator-type and Coulomb-type potentials and their extensions. Although the solutions for those systems could be found by standard methods, this procedure, based on the mapping from the one-dimensional generalized Morse potential via a Langer transformation to the radial Dirac equation in $3+1$ dimensions, provides an easier and powerful way to find the solutions of a class of potentials which otherwise one might not know that would have analytical solutions in the first place. We were able to reproduce well-known particular cases of relativistic harmonic oscillator and Coulomb spin-1/2 systems, when the scalar and vector potentials have the same magnitude, but there are a wealth of other particular cases with physical interest that are left for further study, one of them being solutions with Coulomb-type potentials with tensor components.

\section{Acknowledgements}

This work was supported in part by means of funds provided by CAPES and CNPq (grants 455719/2014-4, 304105/2014-7 and
304743/2015-1). PA would like to thank the Universidade Estadual Paulista, Guaratinguetá Campus, for supporting his stays in its Physics and Chemistry Department and CFisUC for travel support.

\section{References}

[1] C. Tezcan, R. Sever, Int. J. Theor. Phys. 48 (2009) 337.

[2] A.O. Barut, A. Inomata, R. Wilson, J. Math. Phys. 28 (1987) 605.

[3] V.G. Bagrov, D.M. Gitman, Exact Solutions of Relativistic Wave Equations, Kluwer, Dordrecht, 1990.

[4] L. Chetouani, L. Guechi, T.F. Hammann, Helv. Phys. Acta 65 (1992) 1069.

[5] L. Chetouani, L. Guechi, T.F. Hammann, Czechoslov. J. Phys. 43 (1993) 13.

[6] A. Arda, R. Sever, Commun. Theor. Phys. 58 (2012) 27.

[7] L.D. Landau, E.M. Lifshitz, Quantum Mechanics - Non-relativistic Theory, Pergamon, New York, 1958.

[8] I.I. Gol'dman, V.D. Krivchenkov, Problems in Quantum Mechanics, Pergamon, London, 1961.

[9] F. Constantinescu, E. Magyari, Problems in Quantum Mechanics, Pergamon, Oxford, 1971.

[10] D. ter Haar, Problems in Quantum Mechanics, Pion, London, 1975.

[11] C.J. Ballhausen, Chem. Phys. Lett. 146 (1988) 449.

[12] C.J. Ballhausen, Chem. Phys. Lett. 151 (1988) 428.

[13] G. Palm, U. Raff, Am. J. Phys. 71 (2003) 247.

[14] G. Palm, U. Raff, Am. J. Phys. 71 (2003) 956.

[15] S.-H. Dong, M. Louzada-Cassou, Int. J. Mod. Phys. B 19 (2005) 4219.

[16] S.-H. Dong, M. Louzada-Cassou, J. Yu, F. Jiménez-Ángeles, A.L. Rivera, Int. J. Quant. Chem. 107 (2006) 366.

[17] C.A. Singh, O.B. Devi, Int. J. Quant. Chem. 106 (2006) 415.

[18] S.-H. Dong, Factorization Method in Quantum Mechanics, Springer, Dordrecht, 2007.

[19] S.-H. Dong, D. Morales, J. García-Ravelo, Int. J. Mod. Phys. E 16 (2007) 189

[20] S.H. Patil, K.D. Sen, Phys. Lett. A 362 (2007) 109.

[21] S.M. Ikhdair, R. Sever, Int. J. Mod. Phys. C 20 (2009) 361

[22] D.R.M. Pimentel, A.S. de Castro, Rev. Bras. Ensino Fis. 35 (2013) 3303.

[23] E.U. Condon, P.M. Morse, Quantum Mechanics, McGraw-Hill, New York, 1929.

[24] R.L. Hall, N. Saad, J. Chem. Phys. 109 (1998) 2983.

[25] K.J. Oyewumi, Found. Phys. Lett. 18 (2005) 75.

[26] S.M. Ikhdair, Chin. J. Phys. 46 (2008) 291.

[27] D. Agboola, Acta Phys. Pol. 120 (2011) 371.

[28] D.R.M. Pimentel, A.S. de Castro, Rev. Bras. Ensino Fis. 36 (2014) 1307.

[29] T. Das, A. Arda, Adv. High Energy Phys. 2015 (2015) 137038.

[30] P.H.F. Nogueira, A.S. de Castro, J. Math. Chem. 54 (2016) 1783.

[31] R.E. Langer, Phys. Rev. 51 (1937) 669.

[32] M.G. Garcia, A.S. de Castro, L.B. Castro, P. Alberto, Ann. Phys. 378 (2017) 88.

[33] P.H.F. Nogueira, A.S. de Castro, D.R.M. Pimentel, J. Math. Chem. 54 (2016) 1287.

[34] N.N. Lebedev, Special Functions and Their Applications, Dover Publications, New York, 1972.

[35] M. Abramowitz, I. Stegun (Eds.), Handbook of Mathematical Functions, Dover Publications, New York, 1972.

[36] P. Alberto, R. Lisboa, M. Malheiro, A.S. de Castro, Phys. Rev. C 71 (2005) 034313.

[37] H. Liang, J. Meng, S.-G. Zhou, Phys. Rep. 570 (2015) 1.

[38] M. Hamzavi, A.A. Rajabi, H. Hassanabadi, Int. J. Mod. Phys. A 26 (2011) 1363

[39] R. Lisboa, M. Malheiro, A.S. de Castro, P. Alberto, M. Fiolhais, Phys. Rev. C 69 (2004) 024319.

[40] H. Akcay, C. Tezcan, Int. J. Mod. Phys. C 20 (2009) 931.

[41] S. Zarrinkamar, A.A. Rajabi, H. Hassanabadi, Ann. Phys. 325 (2010) 2522.

[42] O. Aydogdu, R. Sever, Few-Body Syst. 47 (2010) 193.

[43] S.M. Ikhdair, R. Sever, J. Math. Phys. 52 (2011) 122108.

[44] G. Chen Gang, Z.-D. Chen, Z.-M. Lou, Chin. Phys. Lett. 13 (2004) 279.

[45] H. Akcay, J. Phys. A 40 (2007) 6427.

[46] V.I. Kukulin, G. Loyola, M. Moshinsky, Phys. Lett. A 158 (1991) 19.

[47] M. Hamzavi, A.A. Rajabi, H. Hassanabadi, Phys. Lett. A 374 (2010) 4303.

[48] M. Hamzavi, A.A. Rajabi, H. Hassanabadi, Few-Body Syst. 48 (2010) 171.

[49] M. Eshghia, S.M. Ikhdair, Chin. Phys. B 23 (2014) 120304.

[50] A.S. de Castro, P. Alberto, Phys. Rev. A 86 (2012) 032122. 